\title{
Dominique Darmaun
}

\section{ADRESSE}

D. Darmaun : professeur associé de pédiatrie. Nemours Children's Clinic, University of Florida, Stable Isotope Lab, 807 Nira Strect, Jacksonville, Florida 32207, États-Unis.

TIRÉS A PART

D. Darmaun.

\section{Intestin et métabolisme de la glutamine}

L'intestin, longtemps considéré comme un organe consacré exclusivement à l'absorption, joue un rôle majeur dans le métabolisme de la glutamine, l'acide aminé le plus abondant chez l'homme. Le métabolisme énergétique intestinal repose pour 30 à $50 \%$ sur l'oxydation de la glutamine. La glutamine a d'autres fonctions dans l'intestin : c'est le précurseur des purines et des pyrimidines, fonction essentielle dans un tissu à taux de renouvellement cellulaire élevé ; c'est le composé énergétique préféré du tissu lymphoïde associé à l'intestin ; c'est, enfín, un puissant stimulant de l'absorption intestinale de l'eau et du sodium. Le rôle thérapeutique éventuel de la glutamine mérite donc d'être mieux étudié, notamment en nutrition humaine.

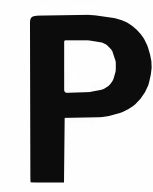

arce qu'elle peut être synthétisée in vivo, la glutamine est classée parmi les acides aminés non essentiels. Elle joue cependant des rôles physiologiques majeurs : (1) dans l'homéostasie protéique comme première navette de l'azote $\alpha$-aminé dans l'organisme, régulateur potentiel de la synthèse protéique et précurseur d'urée ; (2) dans la lutte contre l'acidose en tant que principale source de l'ammonium urinaire ; (3) dans les multiplications cellulaires et l'immunité comme précurseur de bases puriques et pyrimidiques ; (4) enfin, comme carburant privilégié de l'intestin, un rôle de découverte plus récente. Il paraît utile de resituer brièvement la place de la glutamine dans le métabolisme protéino-énergétique avant d'aborder les interrelations entre glutamine et fonctions intestinales chez l'animal, puis les données obtenues in vivo chez l'homme.

\section{Muscle et réserves endogènes de glutamine}

Bien qu'elle représente à peine $6 \%$ des résidus acides aminés des protéines, la glutamine (figure 1) est l'acide $\alpha$-aminé libre le plus abondant de l'organisme [1]. Sa concentration dépasse celle des autres acides $\alpha$ aminés tant dans le plasma (environ $600 \mu \mathrm{M})$ que dans les tissus, et atteint environ 20 millimoles par litre d'eau intracellulaire dans le muscle, où elle constitue les deux tiers du pool intracellulaire d'acides aminés libres 
[1]. Comme la masse musculaire représente $40 \%$ du poids corporel, le contenu en glutamine du corps entier atteint 80 grammes chez l'homme adulte, une masse considérable, du même ordre que le glycogène hépatique (environ $120 \mathrm{~g}$ ). Les maladies graves réduisent parallèlement la capacité de synthèse protéique et la teneur musculaire en glutamine [2] et la restauration du pool de glutamine s'accompagne d'une synthèse protéique accrue [1-4]. Cette corrélation frappante entre taille du pool de glutamine libre et vitesse de synthèse protéique n'existe pour aucun autre acide aminé, et suggère que la glutamine pourrait participer à la régulation de l'homéostasie protéique. Ce rôle - dont le mécanisme reste obscur - n'est qu'une des multiples fonctions attribuées à la glutamine (Tableau I)

\section{La source endogène de glutamine}

Lorsque la lumière de l'intestin est vide de tout aliment, après une nuit de jeûne (phase post-absorptive des auteurs de langue anglaise), le muscle squelettique " exporte " des quantités massives d'acides aminés. Bien que la protéolyse s'accélère à jeun, la composition de ce "débit musculaire " d'acides aminés est loin d'ĉtre celle d'un hydrolysat de protéines : constituant à elles deux moins de $15 \%$ des résidus d'acides aminés des protéines, alanine et glutamine représentent ensemble plus de $60 \%$ des acides aminés libérés par le muscle [1, 4-6]. Aussi l'écrasante majorité de l'alanine et de la glutamine libérées par le muscle doivent-elles provenir d'une synthc̀se de novo. Les acides aminés ramifiés (leucine, isoleucine et valine), qui sont métabolisés dans le muscle, sont les précurseurs probables de cette synthèse (figure 2). Si l'on perfuse de la leucine marquée à l'azote 15 chez un sujet à jeun, on voit vite apparaître le ${ }^{15} \mathrm{~N}$ dans la glutamine et l'alanine circulantes : la transamination leucine-glutamine représente $20 \%$ du flux d'azote de la leucine, et au moins $9 \%$ de l'azote $\alpha$-aminé de la glutamine in vivo [7] ; à eux trois, les acides aminés ramifiés pourraient fournir près du tiers de l'azote de la glutamine.

\section{Flux inter-organes de glutamine}

La protéolyse peut être estimée au niveau du corps entier par la perfusion intraveineuse de traceurs d'un acide aminé indispensable comme la leucine ou la phénylalanine, qui, en phase de jeûne, proviennent exclusivement de la dégradation protéique $[4,7,8]$. La mesure simultanée du débit de renouvellement (turnover) de la glutamine - par perfusion intraveineuse de glutamine marquée - confirme que les deux tiers du débit de glutamine proviennent non pas de la protéolyse, mais d'une synthèse de novo [4]. Le débit interorganes de glutamine $350 \mu \mathrm{mol} . \mathrm{kg}^{-1} \cdot \mathrm{h}^{-1}$ ou $86 \mathrm{~g} / \mathrm{jour}$ chez un adulte de $70 \mathrm{~kg}$ - est le plus

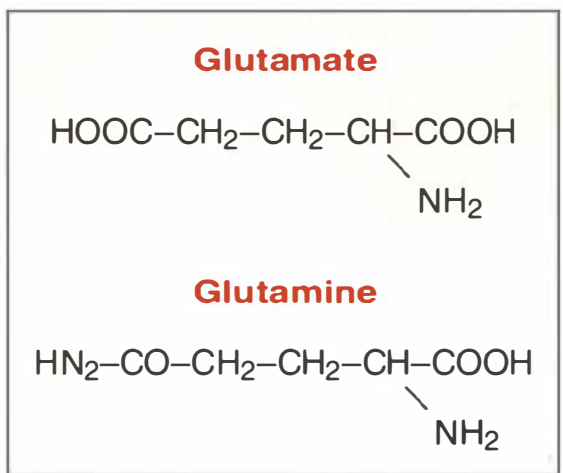

Figure 1. Structure comparée de la glutamine et de l'acide glutamique (glutamate). La seule différence est la présence sur la glutamine d'un second groupement azoté (amide) qui est assez labile in vitro, compliquant à la fois l'analyse de la glutamine et son utilisation en nutrition. rapide de tous les acides aminés étudiés à ce jour, et représente environ la moitié de la production endogène de glucose (Tableau II).

\section{Les " organes cibles" de la glutamine chez I'animal}

On sait depuis plus de trente ans que les deux tiers de l'ammonium excrété proviennent de la fonction amide de la glutamine $[4,9]$. Si la glutamine joue ainsi un rôle critique dans la défense contre l'acidose, la captation rénale de glutamine représente pourtant moins de $10 \%$ du débit de renouvellement de glutamine au niveau du corps entier chez l'homme sain [9].

Au contraire, le rôle de l'intestin

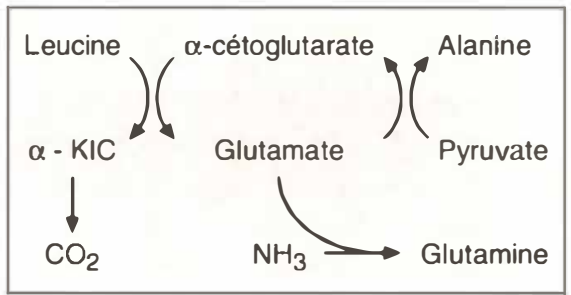

Figure 2. Interrelations entre leucine et glutamine. In vitro, la transamination des acides aminés ramifiés avec I' $\alpha$-cétoglutarate produit un cétoacide II' $\alpha$-céto-isocaproate ou KIC pour la leucinel et de l'acide glutamique, lequel peut alors soit donner son azote au pyruvate pour former de I'alanine, soit accepter un second groupement azoté pour produire de la glutamine.

Tableau I

\section{RÓLES POTENTIELS DE LA GLUTAMINE CHEZ L'HOMME}

- Constituant des protéines (membranaires, enzymatiques, transporteurs, etc.)

- Précurseur de l'ammoniogenèse rénale

- Précurseur de l'uréogenèse hépatique

- Donneur d'azote pour la synthèse des bases puriques et pyrimidiques

- Précurseur de neuromédiateurs (acide glutamique, acide $\gamma$-amino-butyrique)

- Précurseur de glucose

- Source d'énergie (lymphocytes, macrophages, entérocytes)

- Régulateur de la synthèse protéique 
Tableau II

DÉBITS DE RENOUVELLEMENT COMPARÉS DE DIVERS SUBSTRATS CHEZ L'HOMME SAIN A JEUN

\begin{tabular}{|llc|}
\hline Substrat & Traceur & $\begin{array}{c}\text { Débit } \\
\left(\mu \mathrm{mol} . \mathbf{k g}^{-1} \cdot \mathbf{h}\right.\end{array}$ \\
\hline Glucose & D-[6,6-2 $\left.\mathrm{H}_{2}\right]$ glucose & $660 \pm 60$ \\
Leucine & L-[1-13C]leucine & $120 \pm 14$ \\
Alanine & L-[2-15N]alanine & $230 \pm 42$ \\
Glutamine & L-[2-15N]glutamine & $348 \pm 33$ \\
\hline
\end{tabular}

Données personnelles; moyenne \pm écart-type, sur des groupes de 6-8 sujets étudiés en phase de jeûne physiologique, le matin à jeun, par perfusion de substrats marqués aux isotopes stables.

1. Lacey JM, Wilmore DW. Is glutami a conditionally essential amino-acid? Nut Rev 1990 ; 48 : 297-309.

2. McLennan PA, Brown RA, Rennic MJ A positive relationship between protein synthetic rate and intracellular glutamine concentration in perfused rat skeletal mus clc. FEBS Lett 1987 ; 217 : 187-91

3. Stehle P, Zander J, Mertes $\mathrm{N}$, et al. Effect of parenteral glutamine peptide supplements on muscle glutamine loss and nitrogen balance after major surgery. Lancet 1989 ; $\mathrm{i}: 231-3$

4. Darmaun D. Métabolisme de la glutamine in vivo chez l'homme: implications pour la nutrition artificiclle. Nutr Clin Metab (Paris) $1990 ; 4:$ 203-14.

5. Marliss EB, Aoki 'I'T, Pozcfsky 'I', Most AS, Cahill GF. Muscle and splanchnic glutamine and glutamate metabolism in postaborptive and starved man. J Clin Invest $1971 ; 50: 814-7$

6. Felig P. Amino-acid metabolism in man Annu Rev Biochem 1975; 44 : 933-55.

7. Darmaun D, Déchelotte P. Rolc of leucinc as a precursor of glutamine $\alpha$-amino nitrogen in vivo in humans. Am J Physiol 1991 ; 260 : E326-9

8. Déchelotte P, Darmaun D, Hecketsweiler B, Rongier M, Rigal O, Desjeux JF Absorption and metabolic effects of enterally administered glutamine in humans. $A m, J$ Physiol 1991 ; 260 : G677-82.

9. Golden MHN, Jahour P, Jackson AA Glutamine production rate and its contribution to urinary ammonia in normal man Clin Sci $1982 ; 62$ : 299-305.

10. Neptune EM. Respiration and oxidation of various substrates by ileum in vitro. $A m$ J Physiol 1964 ; 209 : 329-32

11. Windmucller HG. Glutamine utilization by the small intestinc. Adv Enzymol 1982 ; 53 : 201-37.

12. Windmucller HG, Spacth AE. Source and fate of circulating citrullinc. Am J Physiol $1981 ; 241$ : E473-80.

13. Squires EJ, Brosnan JT. Measurements of the turnover rate of glutamine in norma and acidotic rats. Biochem J 1983 ; 210 : 277-80. dans le métabolisme de la glutamine a été longtemps méconnu. Même si le rôle du territoire splanchnique avait été établi pour l'alanine et la glutamine [5], on supposait que la glutamine, comme l'alanine, pouvait être captée par le foic pour participer à la gluconéogenèse. On a, en effet, longtemps considéré que le rôle de l'intestin dans le métabolisme azoté se limitait à l'hydrolyse des protéines et à l'absorption des acides aminés. Dans les années 1960, Neptune observa que, en présence de tissu intestinal in vitro, la glutamine disparaissait du milicu d'incubation plus vite que ne pouvait l'expliquer son hydrolyse spontanéc [10]. Dans les années 1970, l'utilisation intestinale de la glutamine fut établie définitivement par une série d'élégantes études réalisées sur l'intestin isolé et perfusé de rat par Windmueller et Spacth [11, 12]. Chez le rat à jeun, $20 \%$ de la glutamine circulante était extraite au premier passage par l'intestin, une extraction dépassant de loin celle de tout autre acide aminé [11]. La quantité extraite correspond à $15-20 \%$ du débit de renouvellement de glutamine au niveau du corps entier [13]. Cette extraction intestinale a depuis lors été confirmée dans nombre d'espèces : rat adulte ou en développement, lapin, chien, porc et poulet $[11,14$, 15]. L'utilisation de glutamine est maximale dans le duodéno-jéjunum, moins intense dans le côlon [11].

\section{Devenir de la glutamine dans l'entérocyte}

Les deux tiers de la glutamine captée par l'intestin de rat sont oxydés
[11]. Ainsi, la glutamine est avant tout un carburant. Le devenir de la glutamine est très différent de celui capté par l'intestin apparaît intact dans le sang portal (figure 3). Aussi, bien que la captation de glutamine et de glucose soient du même ordre de grandeur, la contribution de la glutamine aux dépenses énergétiques de l'intestin représente-t-elle cinq fois celle du glucose [11]. Le devenir de la glutamine dans l'entérocyte ne dépend pas du pôle par lequel elle est captéc (lumière intestinale ou sang circulant). Bien que ces voies métaboliques n'aient pas été parfaitement établics, le schéma suivant (figure 4) est proposé par Hanson et Par-

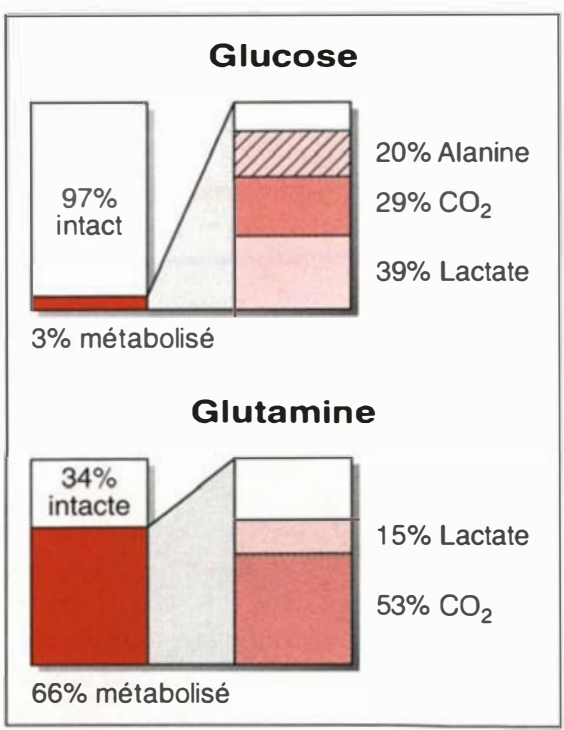

Figure 3. Devenirs comparés du glucose et de la glutamine marqués perfusés dans la lumière du jéjunum de rat (d'après les données de Windmueller et Spaeth (111). du glucose : plus de $90 \%$ du glucose 
sons [16]. La glutamine est hydrolysée en glutamate par une glutaminase intramitochondriale, dont le $\mathrm{Km}$ est 7 fois plus bas que celui de la glutaminase hépatique (ce qui peut rendre compte de l'utilisation de la glutamine dans l'intestin plutôt que dans le foie). Le glutamate serait ensuite non pas désaminé, comme c'est le cas dans le rein, mais transaminé avec du pyruvate, produisant de l'alanine et de l' $\alpha$-cétoglutarate, car l'intest in libère moins de deux moles d'ammonium par mole de glutamine captée (ce qui ne serait pas le cas si le glutamate était désaminé) et les inhibiteurs de l'alanine aminotransférase, comme l'amino-oxyacétate, réduisent la consommation intestinale de glutamine in vitro $[1,11,14] . \mathrm{L}^{\prime} \alpha$ cétoglutarate ainsi produit est ensuite oxydé en acide malique, par une série de réactions faisant partie du cycle de Krebs. L'acide malique serait ensuite décarboxylé par l'enzyme malique en pyruvate; la localisation de l'enzyme impliquée (mitochondriale ou cytoplasmique)

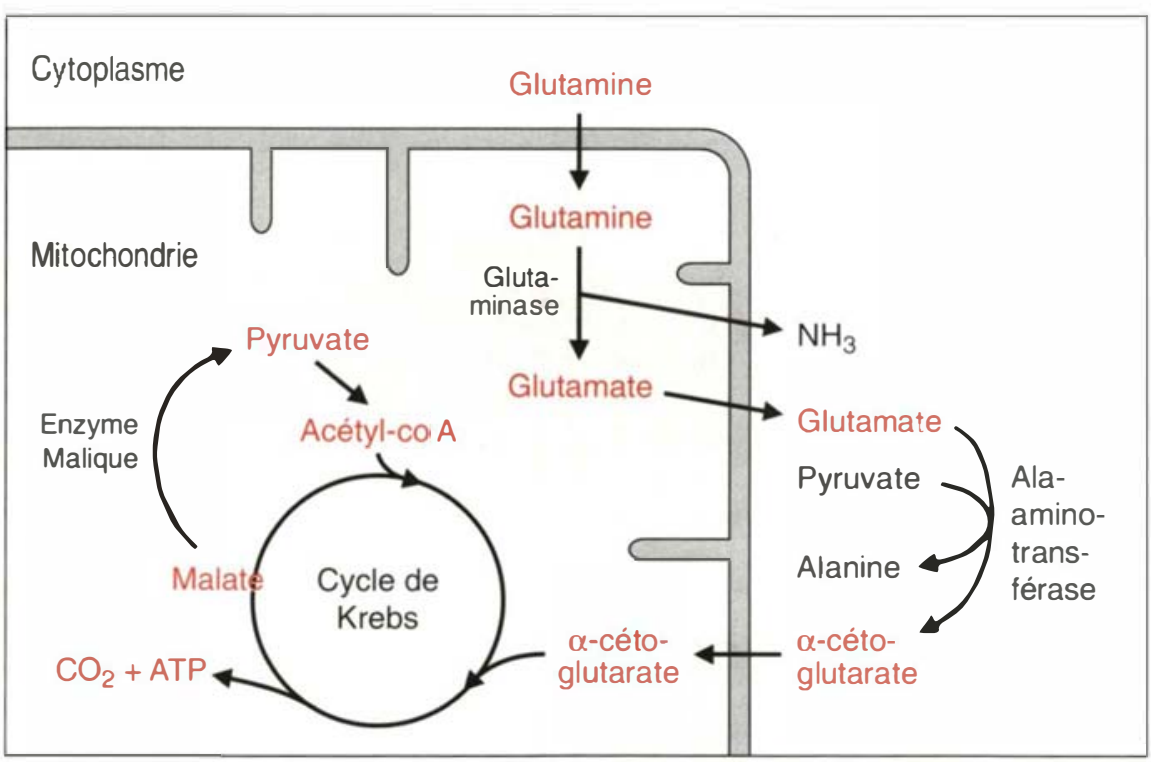

Figure 4. Schéma des principales voies métaboliques de la glutamine dans I'entérocyte (d'après Darmaun [4]).

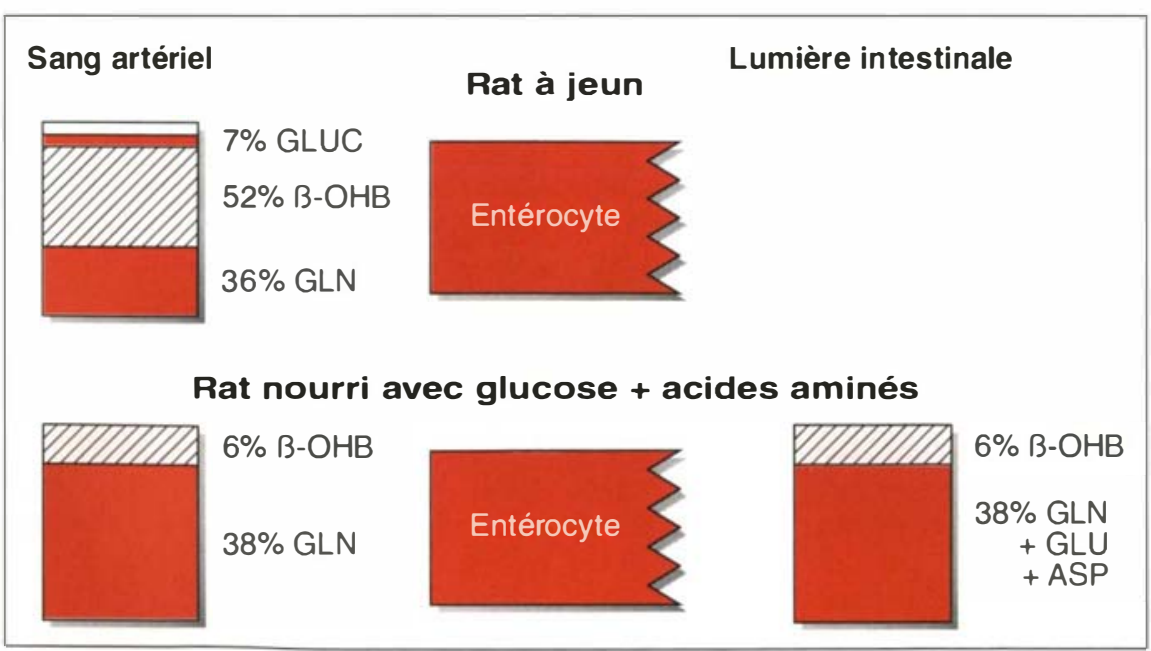

Figure 5. Contribution des divers nutriments aux dépenses énergétiques de l'intestin chez le rat, exprimée en $\%$ de la production totale de $\mathrm{CO}_{2}$. GLUC : glucose ; $\beta$-OHB : corps cétoniques; GLN : glutamine; GLU : glutamate; ASP: aspartate. (D'après les données de Windmueller [11].)

$\mathrm{m} / \mathrm{s} n^{\circ} 8-9$ vol. 9, août-seplembre 93 n'est pas établic. Le pyruvate peut ensuite : (a) soit être converti en acétyl-coenzyme A (sous l'effet de la pyruvate déshydrogénase) pour être " brûlé " dans le cycle de Krebs ; (b), soit accepter un radical aminé et former de l'alanine. Ce schéma explique comment la glutamine pourrait être à la fois un excellent carburant (par sa proximité du cycle de Krebs) et un précurseur de l'azote, voire du squelette carboné de l'alanine produite dans l'intestin. I'intestin est d'ailleurs, après le muscle, le second tissu " exportateur" d'alanine. Quant à l'azote de la glutamine, il se retrouve principalement dans l'ammonium (38\%), mais aussi dans la citrulline (28\%), l'alanine (24\%), la proline et l'ornithine libérées dans la veine porte $[11,14]$. Fn phase de nourriture, l'intestin de rat est capable d'extraire la glutamine tant de la lumière intestinale que du sang circulant. En phase de jeûne, la glutamine fournit $35 \%$, et les corps cétoniques, plus de $50 \%$ de la production de $\mathrm{CO}_{2}$ de l'intestin de rat (figure 5) ; en phase de nourriture, la contribution de la glutamine, du glutamate et de l'aspartate atteindrait plus des deux tiers des dépenses énergétiques de l'intestin ; la moitié de la glutamine viendrait de la lumière intestinale, l'autre moitié du sang artériel [11]. Bien qu'étant un grand utilisateur de glutamine, l'entérocyte est très pauvre en glutamine : la concentration intracellulaire en glutamine y atteint à peine $2 \mathrm{mmol} / \mathrm{kg}$, contre $20 \mathrm{mmol} / \mathrm{kg}$ dans la cellule musculaire [17]. Cela souligne combien l'entérocyte est dépendant de ses "importations" pour la couverture de ses besoins en glutamine.

\section{Métabolisme de la glutamine dans les entérocytes humains}

Les données sur le métabolisme intestinal de la glutamine sont très fragmentaires chez l'homme. In vitro, les entérocytes isolés de l'intestin humain oxydent la glutamine avec la même avidité que ceux du rat; toutefois, alors que les corps cétoniques sont une source majeure d'énergie pour les entérocytes de rat, leur contribution est bien moindre pour les cellu- 


\section{RÉFÉRENCES}

14. Souba WW, Smith RJ, Wilmore IDW. Glutamine metabolism by the intestinal tract. J Parent Ent Nutr 1985; 9 : 608-17.

15. Cersosimo L, PE Williams, PM Radosevitch, et al. Role of glutamine in adaptations in nitrogen metabolism during fasting Am J Physiol 1986 ; 250 : R622-8.

16. Hanson PJ, Parsons DS. The interrelationship between glutamine and alanine in the intestine. Biochem Soc Trans 1980 ; 8 : 506-9.

17. Ollenschlaeger G, Langer K, SchrappeBaecher $\mathrm{M}$, et al. Intestinal glutamine metabolism in patients with HIV-infection. Clin Nutr $1990 ; 9$ : 48-9.

18. Ardawi MSM. Glucose, glutamine and ketone body metabolism in human enterocytes. Metabolism 1988 ; $36: 602-9$.

19. McAnena (J, Moore FA, Jones TN Parsons P. Selective uptake of glutamine in the gastrointestinal tract : confirmation in a human study. Br J Surg 1991; 78 : 480-2.

20. Matthews DE. Utilization of enterally delivered glutamine and glutamate : stable isotope tracer study in healthy man. Clin Nutr $1990 ; 9$ : 50-1.

21. Darmaun D, Messing B, Just B, Rongier M, Desjeux JF. Glutamine metabolism after intestinal resection in humans. Metabolism 1991; $40: 42-4$.

22. Hankard R, Goulet $\mathrm{O}$, Rongier M, et al. A study of glutamine metabolism using stable isotopes in infants with neonatal short bowel syndrome. Clin Nutr 1992; 11 (suppl) : 24 (abstract).

23. Fong $\mathrm{Y}$, Tracey $\mathrm{KJ}$, Hesse $\mathrm{DG}$, et al. Influence of enterectomy on peripheral tissue glutamine efflux in critically ill patients. Surgery 1990 ; 107 : 321-6.

24. Minami H, Morse EL, Adibi SA. Characteristics and mechanism of glutaminedipeptide absorption in human intestine. Gastroenterology 1992 ; 103 : 3-11.

25. Déchelotte $P$. Transport intestinal de la glutamine et effets de la glutamine sur le transport ionique; interrelations avec le métabolisme protéique. Thèse ès Sciences, Université Paris VII, 1992

26. Nath S, Déchelotte $P$, Darmaun D, Gotteland $M$, Rongier M, Desjeux JF. ${ }^{15} \mathrm{~N}$ - and ${ }^{14} \mathrm{C}$-glutamine transport across rabbit ileum in experimental bacterial diar-

\begin{tabular}{|c|c|c|c|c|}
\hline \multicolumn{5}{|c|}{$\begin{array}{c}\text { Tableau III } \\
\text { DIFFÉRENCE ARTÉRIOVEINEUSE DE CONCENTRATION } \\
\text { DE GLUTAMINE ENTRE SANG ARTÉRIEL ET PORTAL CHEZ L'HOMME }\end{array}$} \\
\hline \multirow[b]{2}{*}{$\begin{array}{l}\text { Patients } \\
\text { (référence) }\end{array}$} & \multirow[b]{2}{*}{$\begin{array}{l}\text { Jour } \\
\text { post- } \\
\text { opératoire }\end{array}$} & \multicolumn{2}{|c|}{$\begin{array}{c}\text { Glutamine } \\
\qquad(\mu \mathbf{M})\end{array}$} & \\
\hline & & $\begin{array}{c}\text { Sang } \\
\text { artériel }\end{array}$ & $\begin{array}{c}\text { Sang } \\
\text { portal }\end{array}$ & $\begin{array}{l}\text { Différence } \\
\mu \mathrm{M} \%\end{array}$ \\
\hline Cholécystectomie réglée [6] & 0 & $\begin{array}{l}553 \\
\pm 61\end{array}$ & $\begin{array}{l}460 \\
\pm 48\end{array}$ & $\begin{array}{c}73 \quad 14 \\
\pm \quad 20 \pm 3\end{array}$ \\
\hline Laparotomie exploratrice [19] & $\begin{array}{l}2 \\
5\end{array}$ & $\begin{array}{l}296 \\
\pm 90 \\
292 \\
\pm 69\end{array}$ & $\begin{array}{l}253 \\
\pm 80 \\
255 \\
\pm 69\end{array}$ & $\begin{array}{r}43 \quad 17 \\
\quad \pm 3 \\
37 \quad 13 \\
\pm 3\end{array}$ \\
\hline
\end{tabular}

La concentration plasmatique (moyenne técart-type) de glutamine dans le sang portal a été mesurée soit par ponction peropératoire (jour Ol de la veine porte, soit en post-opératoire après pose d'un cathéter dans la veine porte.

les humaines [18]. Il est vrai que, in vivo, les concentrations circulantes de corps cétoniques sont bien plus faibles chez l'homme que chez le rat.

\section{Apports des méthodes de cathétérisme in vivo chez l'homme}

Dans les années 1970, les différences de concentration mesurées par cathétérisme entre sang artériel et sang veineux sus-hépatique permettaient de conclure que, comme l'alanine, la glutamine est captée essentiellement dans le territoire splanchnique [5]. L'alanine gagne le foie pour entrer dans la gluconéogenèse. Pour faire la part de l'intestin et du foie dans l'utilisation de la glutamine par cette approche, un accès au sang portal est nécessaire, ce qui se heurte à des limites éthiques évidentes. Chez cinq patients opérés pour cholécystectomie, Felig et al. ont observé que le sang prélevé durant l'opération dans la veine porte était appauvri en glutamine par rapport au sang artériel [6]. Plus récemment, McAnena et al. ont implanté des cathéters dans la veine porte de patients ayant subi une laparotomie à la suite d'un traumatisme grave. Ils ont ainsi observé de façon répétée, sur plusieurs jours, une différence de l'ordre de 13 à $17 \%$ dans la concentration de glutamine entre sang artériel et portal [19] (Tableau III). La signification physiologique de données obtenues chez des patients en situation de stress chirurgical ou postopératoire n'est toutefois pas formelle (Tableau IV).

\section{Apport des méthodes isotopiques}

Si l'on perfuse de la ${ }^{15} \mathrm{~N}$-glutamine à dose traceuse chez l'homme sain, alternativement par voie intraveineuse et par voie intragastrique, mais au même débit, l'enrichissement sanguin en traceur atteint un niveau deux fois moindre lors de la perfusion intragastrique, impliquant qu'environ 50-60\% de la glutamine perfusée par voie digestive est extraite dans le territoire splanchnique [20]. A contrario, le "modèle " de la résection intestinale étendue (" homme sans intestin ") a été utilisé afin de mettre en évidence l'importance quantitative de l'intestin dans l'utilisation de glutamine chez l'homme. Chez des patients adultes présentant un grêle très court $(<1,5 \mathrm{~m})$ après résection étendue, plusieurs mois après l'intervention, le débit de renouvellement de glutamine a été trouvé abaissé de $20 \%$ par rapport à des témoins sains, alors que les patients étaient sortis de la phase de stress chirurgical, et dans un état nutritionnel satisfaisant après sevrage de toute nutrition artificielle [21]. Cet abaissement du débit de renouvellement de glutamine n'était pas lié à des perturbations globales du métabolisme protéique : le métabolisme de la leucine 


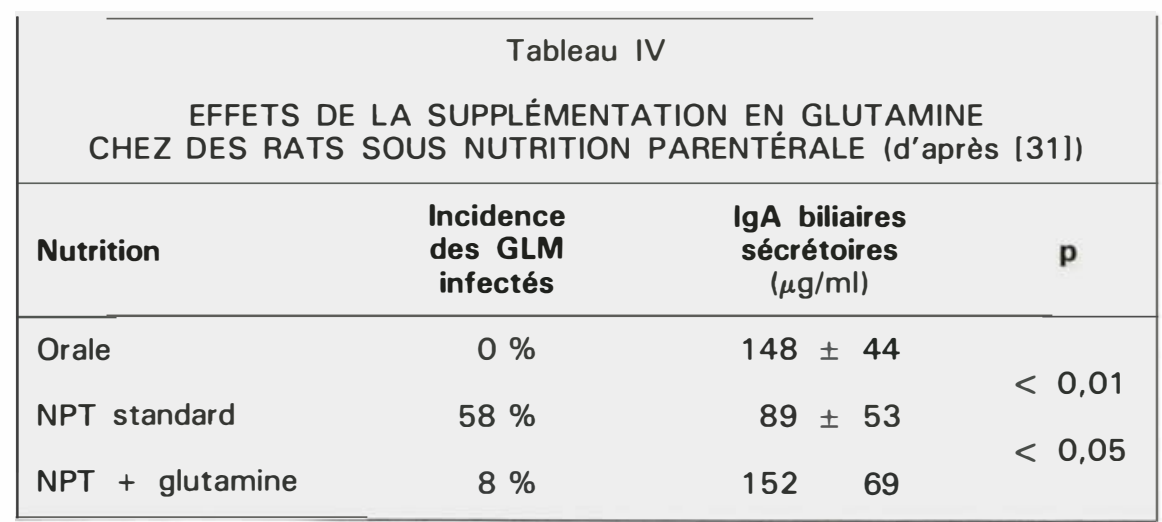

L'incidence des cultures bactériennes positives à partir des ganglions lymphatiques mésentériques (GLM), ainsi que les taux d'immunoglobulines A sécrétoires (IgA) dans la bile ont été déterminés dans trois groupes de rats en nutrition orale et en nutrition parentérale standard (NPT) ou supplémentée en glutamine.

était normal chez ces patients. Le débit de renouvellement de glutamine ayant été mesuré à l'état stationnaire, sa réduction traduit une diminution tant de l'utilisation que de la production endogène de glutamine. Le mécanisme de cette baisse reste à élucider: on peut imaginer que la réduction de la masse intestinale diminue dans un premier temps la consommation intestinale de glutamine et élève la concentration circulante de glutamine; cette élévation de la glutamine plasmatique pourrait ensuite freiner la synthèse musculaire - donc la production endogène de glutamine, ce qui, à long terme, aboutirait à rétablir une concentration normale de glutamine plasmatique. La réduction de l'utilisation de glutamine chez des patients présentant une amputation de leur masse cellulaire intestinale suggère que l'intestin grêle est bien un site majeur d'utilisation de la glutamine in vivo chez l'homme, comme chez l'animal. Des données similaires ont été obtenues dans un groupe de nourrissons entérectomisés [22]. Une étude récente suggère que la production musculaire de glutamine pourrait être réglée par la masse intestinale. La libération de glutamine par le muscle périphérique a été mesurée dans deux groupes de patients de réanimation: l'un des groupes avait subi une résection étendue du grêle, l'autre une simple laparotomie exploratrice. Chez tous les patients, le muscle libérait de la glu-

thérapeutique. La glutamine ne paraît pas affecter la production hépatique de glucose, ni la protéolyse, mais pourrait freiner la lipolyse. En outre, l'apport de glutamine entraîne une élévation de la synthèse endogène d'alanine et de la concentration plasmatique de citrulline, suggérant que la glutamine pourrait être convertie en alanine et citrulline dans l'entérocyte, comme c'est le cas chez le rat. Enfin, une étude récente montre que lorsque des dipeptides contenant de la glutamine (alanyl- ou glycylglutamine) sont apportés par voie luminale, ils sont préférentiellement absorbés intacts, puis hydrolysés secondairement dans la muqueuse intestinale plutôt qu'hydrolysés dans la lumière intestinale [24].

tamine, et tous étaient dans un état de stress comparable, si l'on en juge par les taux circulants de cortisol et de catécholamines. Pourtant, la libération musculaire de glutamine était effondrée chez les patients à grêle court [23].

\section{L'absorption intestinale de la glutamine}

Un régime riche en protéine (100 g/jour) apporte 10 à 20 grammes de glutamine et de glutamate par jour : une quantité importante, même comparée à la production endogène ( $\simeq 80 \mathrm{~g} /$ jour). Bien que la cinétique et les mécanismes de transport de la glutamine soient progressivement élucidés sur des préparations d'entérocytes ou de bordure en brosse isolée [24], l'absorption et les effets de la glutamine apportée par voie intestinale n'ont été quantifiés que récemment in vivo chez l'homme par perfusion jéjunale de L-glutamine naturelle, couplée à la perfusion intraveineuse de traceurs (glucose, alanine et leucine marquées) [8, 25]. L'absorption de la glutamine est très rapide, ses paramètres cinétiques sont voisins de ceux du D-glucose dans le jéjunum humain [8]. L'apport entéral de glutamine, malgré l'extraction splanchnique, entraîne une élévation de la concentration plasmatique de glutamine dépendante de la dose : la voie entérale paraît donc praticable pour administrer de la glutamine en

\section{Pourquoi la glutamine est-elle un carburant privilégié de l'intestin?}

D'un point de vue finaliste, il est légitime de s'interroger sur le " choix " de la glutamine comme carburant privilégié pour l'intestin.

Tout d'abord, la glutamine est un carburant avantageux puisque : (1) de vastes stocks de glutamine sont disponibles dans l'organisme, et renouvelés activement par synthèse de novo (voir plus haut) ; (2) le rendement de l'oxydation de la glutamine (5 moles d'ATP par atome de carbone) est similaire à celui du glucose ; (3) enfin, l'intestin est un site idéal pour l'oxydation de la glutamine, puisque l'ammonium produit par son oxydation peut être libéré sans risque dans la veine porte pour rejoindre directement l'uréogenèse hépatique.

Mais il est clair que dans l'intestin, la glutamine n'est pas seulement un carburant. Elle stimule la fonction princeps de l'épithélium, à savoir l'absorption d'eau et de sodium, tant chez l'animal que chez l'homme, aussi bien à l'état basal que dans plusieurs "modèles" de diarrhée expérimentale $[25,26]$. L'effet de la glutamine sur le transport de sodium n'est pas spécifique de la glutamine - il s'observe avec de nombreux autres substrats, et le traitement des diarrhées par la réhydratation par voie orale est fondé sur des solutions à base de glucose -, mais il est additif par rapport à celui du glucose. 


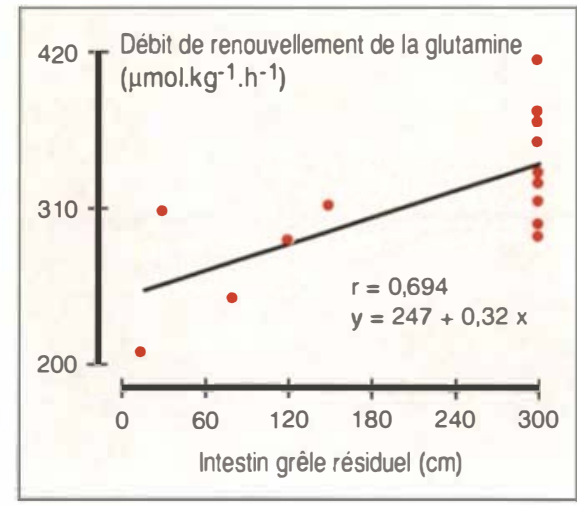

Figure 6. Relation entre la longueur d'intestin grêle résiduel et le débit de renouvellement de glutamine chez I'homme. Si l'on place sur un même graphique les patients à grêle court et les sujets sains - avec une longueur d'intestin arbitrairement estimée à 3 mètres -, on observe une corrélation significative entre la longueur d'intestin grêle et le débit de renouvellement de glutamine (d'après les données de Darmaun et al. [21]).

\section{RÉFÉRENCES}

27. Rexdiger WEW. Metabolic basis of starvation diarrhoea : implications for treatment. Lancet 1986 ; i : 1082-4.

28. Parry-Billings $M$, Evans $J$, Calder PC, Newsholme EA. 1)oes glutamine contribute to immunosuppression after major burns? l.ancet $1990 ; 336$ : 523-5.

29. Fox Al), Kripke SA, 1)e Paula J, et al. Effect of a glutamine-supplemented enteral diet on methotrexate-induced enterocolitis. J Parent Ent Nutr 1988; 12 : 325-31.

30. Souba WW, Klimberg S, Matamaki RI), et al. (Oral glutamine reduces bacterial translocation following abdominal radiation. J Surg Res $1990 ; 48: 1-5$.

31. Burke 1)J, Alverdy JC, Aoys E, Moss GS. Glutamine-supplemented total parenteral nutrition improves gut immune function. Arch Surg 1989; 124: 1396-9.

32. Ziegler 'TR, Young I,S, Benfell K, et al. Climical and metabolic efficacy of glutamine-supplemented parenteral nutrition after bone marrow transplantation. Ann
Bien plus, par rapport au glucose, l'ajout de glutamine aux solutions de réhydratation présenterait l'avantage théorique d'apporter non seulement une stimulation de l'absorption du sodium, mais de l'énergic pour "nourrir" l'intestin lui-même [27].

En outre, il faut rappeler que le " choix " de la glutamine comme carburant privilégié est une caractéristique commune de toutes les cellules à multiplication active, comme les cellules cancéreuses, les fibroblastes en culture et les cellules du système immunitaire, tant macrophages que lymphocytes, quiescents ou activés [1, 4 , 28]. Cela tient probablement au fait que la glutamine est aussi un précurseur dans la synthèse des bases puriques et pyrimidiques, besoin essentiel pour des tissus où la synthèse d'acides nucléiques est active. Ainsi, la glutamine pourrait avoir un effet trophique sur l'épithélium digestif lui-même et/ou le système immunitaire intestinal. Chez l'animal, l'atrophic muqueuse induite par la " mise au repos digestif " pourrait ĉtre prévenue par l'administration de glutamine seule [1]. Chez le rat, l'administration de méthotrexatc produit des lésions d'entérocolite mortelle dans près de $100 \%$ des cas : la supplémentation entérale en glutamine atténue la perte de poids et accroît la trophicité des muqueuses du jéjunum et du côlon, mesurée par la hauteur des cryptes, le nombre des mitoses, ou son contenu en acides nucléiques et en protéines [29] ; l'incidence des bactériémies est réduite, et la survic en est prolongée [1]. Un déficit en glutamine a été évoqué pour expliquer l'augmentation anormale de la perméabilité intestinale aux bactéries lors de l'agression chirurgicale ou de la radiothérapie : l'apport de glutamine réduit la fréquence de contamination bactérienne des ganglions lymphatiques mésentériques et le passage sanguin des bactéries intestinales chez le rat irradié $[30]$ ou en nutrition parentérale [31]. Chez des patients subissant une greffe de moelle osseuse pour hémopathie maligne, la supplémentation en glutamine de l'alimentation parentérale semble réduire la fréquence des infections à point de départ intestinal [32]. Enfin, le métabolisme intestinal de la glutamine pourrait servir de source de citrulline, qui échappe au foie pour être convertic en arginine par le rein ; l'arginine produite pourrait ensuite jouer un rôle immunostimulant comme source d'oxyde nitrique dans les macrophages.

En conclusion, ce rapide aperçu des relations entre intestin et glutamine laisse entrevoir non seulement un nouveau chapitre de physiologic concernant une fonction longtemps méconnue de l'intestin, son rôle métabolique, mais aussi de vastes perspectives d'utilisation de la glutamine en thérapeutique, notamment nutritionnelle [1, 4]

\section{Summary}

Glutamine metabolism in the gut

Although long considered almost exclusively as an absorptive organ, the gut plays a prominent role in the metabolism of glutamine, the most abundant amino acid in the body. The small intestine extracts more than $20 \%$ of whole body glutamine production in most species, and, most likely, humans as well. Glutamine oxidation provides $30-50 \%$ of the energy requirement of the gut, 5 times the contribution of glucose. Glutamine's use as a preferred intestinal fuel seems advantageous in view of : (1) the large $(\simeq 80 \mathrm{~g})$, and continuously replenished $(\simeq 85 \mathrm{~g} / \mathrm{d})$ glutamine stores available in the body ; (2) the role of glutamine as a precursor of purines and pyrimidines, the building blocks of nucleic acids, that are in high demand in a tissue with high rates of cell division; (3) the role of glutamine as a preferred fuel for the gut-associated lymphoid tissue ; and (4) finally, glutamine's potent stimulatory effect on sodium and water absorption. The multifaceted role of glutamine in the small intestine suggests further evaluation of its potential therapeutic use is warranted. 